\title{
DistDGL: Distributed Graph Neural Network Training for Billion-Scale Graphs
}

\author{
Da Zheng \\ AWS AI \\ dzzhen@amazon.com \\ Xiang Song \\ AWS Shanghai AI Lab \\ xiangsx@amazon.com
}

\author{
Chao Ma \\ AWS Shanghai AI Lab \\ manchao@amazon.com
}

\author{
Quan Gan \\ AWS Shanghai AI Lab \\ quagan@amazon.com
}

\author{
Minjie Wang \\ AWS Shanghai AI Lab \\ minjiw@amazon.com
}

\author{
Jinjing Zhou \\ AWS Shanghai AI Lab \\ zhoujinj@amazon.com
}

\author{
Qidong $\mathrm{Su}$ \\ AWS Shanghai AI Lab \\ qidos@amazon.com
}

\author{
Zheng Zhang \\ AWS Shanghai AI Lab \\ zhaz@amazon.com
}

\author{
George Karypis \\ AWS AI \\ gkarypis@amazon.com
}

\begin{abstract}
Graph neural networks (GNN) have shown great success in learning from graph-structured data. They are widely used in various applications, such as recommendation, fraud detection, and search. In these domains, the graphs are typically large, containing hundreds of millions of nodes and several billions of edges. To tackle this challenge, we develop DistDGL, a system for training GNNs in a mini-batch fashion on a cluster of machines. DistDGL is based on the Deep Graph Library (DGL), a popular GNN development framework. DistDGL distributes the graph and its associated data (initial features and embeddings) across the machines and uses this distribution to derive a computational decomposition by following an ownercompute rule. DistDGL follows a synchronous training approach and allows ego-networks forming the mini-batches to include non-local nodes. To minimize the overheads associated with distributed computations, DistDGL uses a high-quality and lightweight min-cut graph partitioning algorithm along with multiple balancing constraints. This allows it to reduce communication overheads and statically balance the computations. It further reduces the communication by replicating halo nodes and by using sparse embedding updates. The combination of these design choices allows DistDGL to train high-quality models while achieving high parallel efficiency and memory scalability. We demonstrate our optimizations on both inductive and transductive GNN models. Our results show that DistDGL achieves linear speedup without compromising model accuracy and requires only 13 seconds to complete a training epoch for a graph with 100 million nodes and 3 billion edges on a cluster with 16 machines. DistDGL is now publicly available as part of DGL: https://github.com/dmlc/dgl/tree/master/python/dgl/distributed.

Index Terms-
\end{abstract}

\section{INTRODUCTION}

Graph Neural Networks (GNNs) have shown success in learning from graph-structured data and have been applied to many graph applications in social networks, recommendation, knowledge graphs, etc. In these applications, graphs are usually huge, in the order of many millions of nodes or even billions of nodes. For instance, Facebook social network graph contains billions of nodes. Amazon is selling billions of items and has billions of users, which forms a giant bipartite graph for its recommendation task. Natural language processing tasks take advantage of knowledge graphs, such as Freebase [1] with 1.9 billion triples.
It is challenging to train a GNN model on a large graph. Unlike domains such as computer vision and natural language processing, where training samples are mutually independent, graph inherently represents the dependencies among training samples (i.e., vertices). Hence, mini-batch training on GNNs is different from the traditional deep neural networks; each mini-batch must incorporate those depending samples. The number of depending samples usually grows exponentially when exploring more hops of neighbors. This leads to many efforts in designing various sampling algorithms to scale GNNs to large graphs [2]-[6]. The goal of these methods is to prune the vertex dependency to reduce the computation while still estimating the vertex representation computed by GNN models accurately.

It gets even more challenging to train GNNs on giant graphs when scaling beyond a single machine. For instance, a graph with billions of nodes requires memory in the order of terabytes attributing to large vertex features and edge features. Due to the vertex dependency, distributed GNN training requires to read hundreds of neighbor vertex data to compute a single vertex representation, which accounts for majority of network traffic in distributed GNN training. This is different from traditional distributed neural network training, in which majority of network traffic comes from exchanging the gradients of model parameters. In addition, neural network models are typically trained with synchronized stochastic gradient descent (SGD) to achieve good model accuracy. This requires the distributed GNN framework to generate balanced mini-batches that contain roughly the same number of nodes and edges as well as reading the same account of data from the network. Due to the complex subgraph structures in natural graphs, it is difficult to generate such balanced mini-batches.

Unfortunately, current systems cannot effectively address the challenges of distributed GNN training. Previous distributed graph analytical systems [7]-[9] are designed for full graph computation expressed in the vertex-centric program paradigm, which is not suitable for GNN mini-batch training. Existing domain-specific frameworks for training GNNs, such as DGL [10] and PyTorch-Geometric [11], cannot scale to giant graphs. They were mainly developed for training on a 
single machine. Although there have been some efforts in building systems for distributed GNN training, they either focus on full batch training by partitioning graphs to fit the aggregated memory of multiple devices [12]-[14] or suffer from the huge network traffic caused by fetching neighbor node data [15]-[17]. System architectures [18]-[20] proposed for training neural networks for computer vision and natural language processing are not directly applicable because one critical bottleneck in GNN training is the network traffic of fetching neighbor node data due to the vertex dependencies, while previous systems majorly focuses on network traffic from exchanging the gradients of model parameters.

In this work, we develop DistDGL on top of DGL to perform efficient and scalable mini-batch GNN training on a cluster of machines. It provides distributed components with APIs compatible to DGL's existing ones. As such, it requires trivial effort to port DGL's training code to DistDGL. Internally, it deploys multiple optimizations to speed up computation. It distributes graph data (both graph structure and the associated data, such as node and edge features) across all machines and run trainers, sampling servers (for sampling subgraphs to generate mini-batches) and in-memory KVStore servers (for serving node data and edge data) all on the same set of machines. To achieve good model accuracy, DistDGL follows a synchronous training approach and allows ego-networks forming the mini-batches to include non-local nodes. To reduce network communication, DistDGL adopts METIS [21] to partition a graph with minimum edge cut and co-locate data with training computation. In addition, DistDGL deploys multiple load balancing optimizations to tackle the imbalance issue, including multi-constraint partitioning and two-level workload splitting. DistDGL further reduces network communication in sampling by replicating halo nodes in the partitioned graph structure but does not replicate data in halo nodes to have a small memory footprint. DistDGL provides distributed embeddings with efficient sparse updates for transductive graph models.

We conduct comprehensive experiments to evaluate the efficiency of DistDGL and effectiveness of the optimizations. Overall, DistDGL achieves $2.2 \times$ speedup over Euler on a cluster of four CPU machines. The main performance advantage comes from the efficient feature copy with $5 \times$ data copy throughput. DistDGL speeds up the training linearly without compromising model accuracy as the number of machines increases in a cluster of 16 machines and easily scales the GraphSage model to a graph with 100 million nodes and 3 billion edges. It takes 13 seconds per epoch to train on such a graph in a cluster of 16 machines.

\section{BACKGROUND}

\section{A. Graph Neural Networks}

GNNs emerge as a family of neural networks capable of learning a joint representation from both the graph structure and vertex/edge features. Recent studies [22], [23] formulate GNN models with message passing, in which vertices broadcast messages to their neighbors and compute their own representation by aggregating received messages.

More formally, given a graph $\mathcal{G}(\mathcal{V}, \mathcal{E})$, we denote the input feature of vertex $v$ as $\mathbf{h}_{v}^{(0)}$, and the feature of the edge between vertex $u$ and $v$ as $\mathbf{e}_{u v}$. To get the representation of a vertex at layer $l$, a GNN model performs the computations below:

$$
\mathbf{h}_{v}^{(l+1)}=g\left(\mathbf{h}_{v}^{(l)}, \bigoplus_{u \in \mathcal{N}(v)} f\left(\mathbf{h}_{u}^{(l)}, \mathbf{h}_{v}^{(l)}, \mathbf{e}_{u v}\right)\right)
$$

Here $f, \bigoplus$ and $g$ are customizable or parameterized functions (e.g., neural network modules) for calculating messages, aggregating messages, and updating vertex representations, respectively. Similar to convolutional neural networks (CNNs), a GNN model iteratively applies Equations (1) to generate vertex representations for multiple layers.

There are potentially two types of model parameters in graph neural networks. $f, \bigoplus$ and $g$ can contain model parameters, which are shared among all vertices. These model parameters are updated in every mini-batch and we refer to these parameters as dense parameters. Some GNN models may additionally learn an embedding for each vertex. Embeddings are part of the model parameters and only a subset of vertex embeddings are updated in a mini-batch. We refer to these model parameters as sparse parameters.

\section{B. Mini-batch training}

GNN models on a large dataset can be trained in a minibatch fashion just like deep neural networks in other domains like computer vision and natural language processing. However, GNN mini-batch training is different from other neural networks due to the data dependency between vertices. Therefore, we need to carefully sample subgraphs that capture the data dependencies in the original graph to train GNN models.

A typical strategy of training a GNN model [2] follows three steps: (i) sample a set of $N$ vertices, called target vertices, uniformly at random from the training set; (ii) randomly pick at most $K$ (called fan-out) neighbor vertices for each target vertex; (iii) compute the target vertex representations by gathering messages from the sampled neighbors. When the GNN has multiple layers, the sampling is repeated recursively. That is, from a sampled neighbor vertex, it continues sampling its neighbors. The number of recursions is determined by the number of layers in a GNN model. This sampling strategy forms a computation graph for passing messages on. Figure $1 \mathrm{~b}$ depicts such a graph for computing representation of one target vertex when the GNN has two layers. The sampled graph and together with the extracted features are called a mini-batch in GNN training.

There have been many works regarding to the different strategies to sample graphs for mini-batch training [3], [4], [24]-[26]. Therefore, a GNN framework needs to be flexible as well as scalable to giant graphs. 


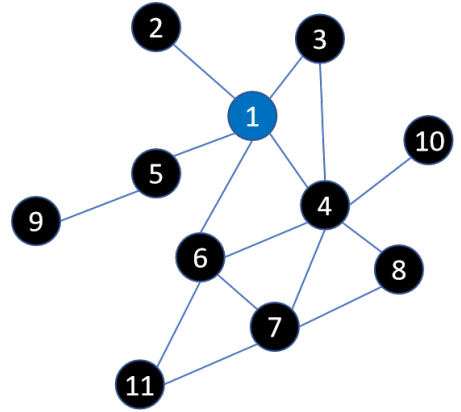

(a) An input graph.

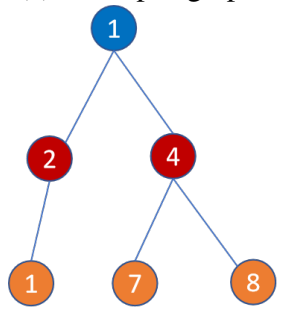

(b) A sampled graph for computing one target vertex representation with a two-layer GNN model. Messages flow from leaves to root.

Fig. 1: One sampled mini-batch in GNN training.

\section{DistDGL SyStem Design}

\section{A. Distributed Training Architecture}

DistDGL distributes the mini-batch training process of GNN models to a cluster of machines. It follows the synchronous stochastic gradient descent (SGD) training; each machine computes model gradients with respect to its own mini-batch, synchronizes gradients with others and updates the local model replica. At a high level, DistDGL consists of the following logical components (Figure 2):

- A number of samplers in charge of sampling the minibatch graph structures from the input graph. Users invoke DistDGL samplers in the trainer process via the same interface in DGL for neighbor sampling, which internally becomes a remote process call (RPC). After mini-batch graphs are generated, they are sent back to the trainers.

- A KVStore that stores all vertex data and edge data distributedly. It provides two convenient interfaces for pulling the data from or pushing the data to the distributed store. It also manages the vertex embeddings if specified by the user-defined GNN model.

- A number of trainers that compute the gradients of the model parameters over a mini-batch. At each iteration, they first fetch the mini-batch graphs from the samplers and the corresponding vertex/edge features from the KVStore. They then run the forward and backward computation on their own mini-batches in parallel to compute the gradients. The gradients of dense parameters are dispatched to the dense model update component for synchronization, while the gradients of sparse embeddings are sent back to the KVStore for update.

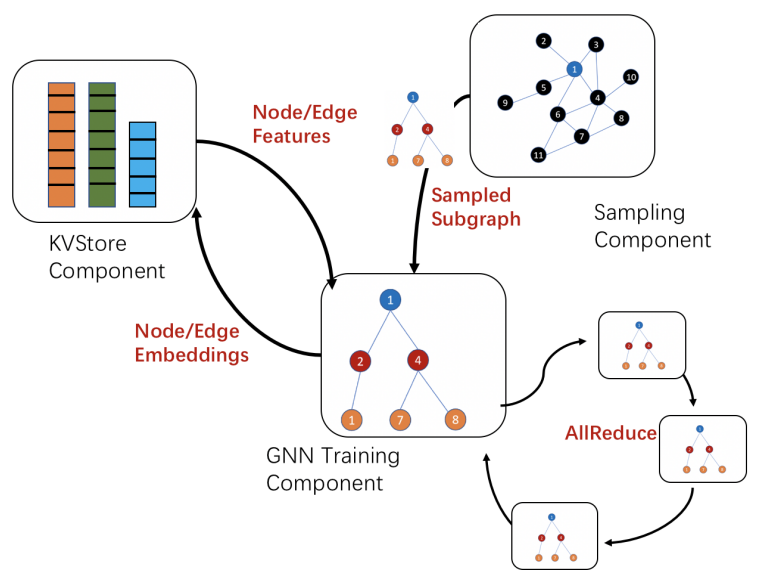

Fig. 2: DistDGL's logical components.

- A dense model update component for aggregating dense GNN parameters to perform synchronous SGD. DistDGL reuses the existing components depending on DGL's backend deep learning frameworks (e.g., PyTorch, MXNet and TensorFlow). For example, DistDGL calls the all-reduce primitive when the backend framework is PyTorch [27], or resorts to parameter servers [18] for MXNet and TensorFlow backends.

When deploying these logical components to actual hardware, the first consideration is to reduce the network traffic among machines because graph computation is data intensive [28]. DistDGL adopts the owner-compute rule (Figure 3). The general principle is to dispatch computation to the data owner to reduce network communication. DistDGL first partitions the input graph with a light-weight min-cut graph partitioning algorithm. It then partitions the vertex/edge features and co-locates them with graph partitions. DistDGL launches the sampler and KVStore servers on each machine to serve the local partition data. Trainers also run on the same cluster of machines and each trainer is responsible for the training samples from the local partition. This design leverages data locality to its maximum. Each trainer works on samples from the local partition so the mini-batch graphs will contain mostly local vertices and edges. Most of the mini-batch features are locally available too via shared memory, reducing the network traffic significantly. In the following sections, we will elaborate more on the design of each components.

\section{B. Graph Partitioning}

The goal of graph partitioning is to split the input graph to multiple partitions with a minimal number of edges across partitions. Graph partitioning is a preprocessing step before distributed training. A graph is partitioned once and used for many distributed training runs, so its overhead is amortized.

DistDGL adopts METIS [21] to partition a graph. This algorithm assigns densely connected vertices to the same partition to reduce the number of edge cuts between partitions (Figure 4a). After assigning some vertices to a partition, DistDGL assigns all incident edges of these vertices to the 


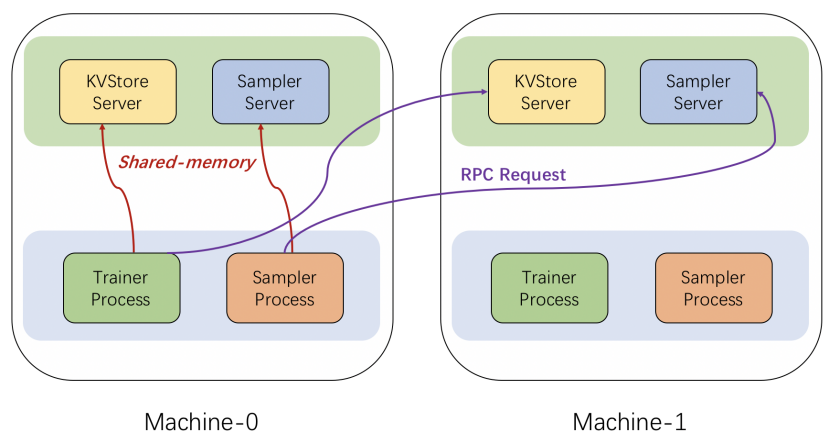

Fig. 3: The deployment of DistDGL's logical components on a cluster of two machines.

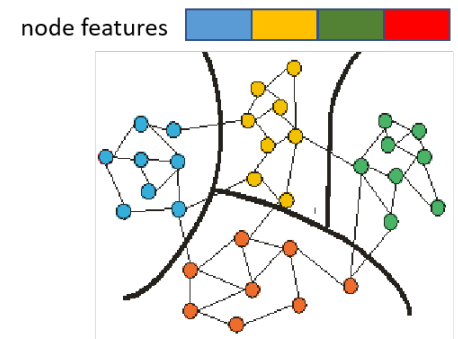

(a) Assign vertices to graph partitions

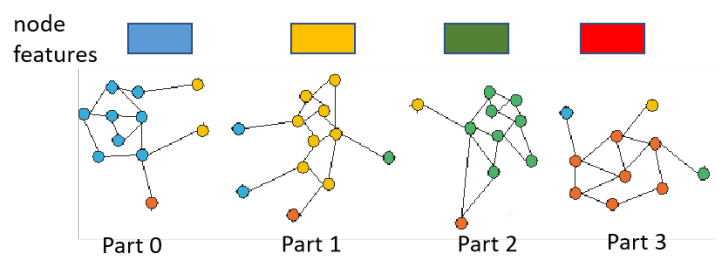

(b) Generate graph partitions with HALO vertices (the vertices with different colors from majority of the vertices in the partition).

Fig. 4: Graph partitioning with METIS in DistDGL.

same partition. This ensures that all the neighbors of the local vertices are accessible on the partition so that samplers can compute locally without communicating to each other. With this partitioning strategy, each edge has a unique assignment while some vertices may be duplicated (Figure 4b). We refer to the vertices assigned by METIS to a partition as core vertices and the vertices duplicated by our edge assignment strategy as $H A L O$ vertices. All the core vertices also have unique partition assignments.

While minimizing edge cut, DistDGL deploys multiple strategies to balance the partitions so that mini-batches of different trainers are roughly balanced. By default, METIS only balances the number of vertices in a graph. This is insufficient to generate balanced partitions for synchronous minibatch training, which requires the same number of batches from each partition per epoch and all batches to have roughly the same size. We formulate this load balancing problem as a multi-constraint partitioning problem, which balances the partitions based on user-defined constraints [29]. DistDGL takes advantage of the multi-constraint mechanism in METIS to balance training/validation/test vertices/edges in each partition as well as balancing the vertices of different types and the edges incident to the vertices of different types.

METIS' partitioning algorithms are based on the multilevel paradigm, which has been shown to produce high-quality partitions. However, for many types of graphs involved in learning on graphs tasks (e.g., graphs with power-law degree distribution), the successively coarser graphs become progressively denser, which considerably increases the memory and computational complexity of multilevel algorithms. To address this problem, we extended METIS to only retain a subset of the edges in each successive graph so that the degree of each coarse vertex is the average degree of its constituent vertices. This ensures that as the number of vertices in the graph reduces by approximately a factor of two, so do the edges. To ensure that the partitioning solutions obtained in the coarser graphs represent high-quality solutions in the finer graphs, we only retain the edges with the highest weights in the coarser graph. In addition, to further reduce the memory requirements, we use an out-of-core strategy for the coarser/finer graphs that are not being processed currently. Finally, we run METIS by performing a single initial partitioning (default is 5) and a single refinement iteration (default is 10) during each level. For power-law degree graphs, this optimization leads to a small increase in the edge-cut (2\%-10\%) but considerably reduces its runtime. Overall, the set of optimizations above compute high-quality partitionings requiring $5 \times$ less memory and $8 \times$ less time than METIS' default algorithms.

After partitioning the graph structure, we also partition vertex features and edge features based on the graph partitions. We only assign the features of the core vertices and edges of a partition to the partition. Therefore, the vertex features and edge features are not duplicated.

After graph partitioning, DistDGL manages two sets of vertex IDs and edge IDs. DistDGL exposes global vertex IDs and edge IDs for model developers to identify vertices and edges. Internally, DistDGL uses local vertex IDs and edge IDs to locate vertices and edges in a partition efficiently, which is essential to achieve high system speed as demonstrated by previous works [30]. To save memory for maintaining the mapping between global IDs and local IDs, DistDGL relabels vertex IDs and edge IDs of the input graph during graph partitioning to ensure that all IDs of core vertices and edges in a partition fall into a contiguous ID range. In this way, mapping a global ID to a partition is binary lookup in a very small array and mapping a global ID to a local ID is a simple subtraction operation.

\section{Distributed Key-Value Store}

The features of vertices and edges are partitioned and stored in multiple machines. Even though DistDGL partitions a graph to assign densely connected vertices to a partition, we still need to read data from remote partitions. To simplify the data access on other machines, DistDGL develops a distributed inmemory key-value store (KVStore) to manage the vertex and edge features as well as vertex embeddings, instead of using an existing distributed in-memory KVStore, such as Reddis, 
for (i) better co-location of node/edge features in KVStore and graph partitions, (ii) faster network access for high-speed network, (iii) efficient updates on sparse embeddings.

DistDGL's KVStore supports flexible partition policies to map data to different machines. For example, vertex data and edge data are usually partitioned and mapped to machines differently as shown in Section III-B DistDGL defines separate partition policies for vertex data and edge data, which aligns with the graph partitions in each machine.

Because accessing vertex and edge features usually accounts for the majority of communication in GNN distributed training, it is essential to support efficient data access in KVStore. A key optimization for fast data access is to use shared memory. Due to the co-location of data and computation, most of data access to KVStore results in the KVStore server on the local machine. Instead of going through Inter-Process Communication (IPC), the KVStore server shares all data with the trainer process via shared memory. Thus, trainers can access most of the data directly without paying any overhead of communication and process/thread scheduling. We also optimize network transmission of DistDGL's KVStore for fast networks (e.g., 100Gbps network). We develop an optimized RPC framework for fast networking communication, which adopts zero-copy mechanism for data serialization and multithread send/receive interface.

In addition to storing the feature data, we design DistDGL's KVStore to support sparse embedding for training transductive models with learnable vertex embeddings. Examples are knowledge graph embedding models [31]. In GNN mini-batch training, only a small subset of vertex embeddings are involved in the computation and updated during each iteration. Although almost all deep learning frameworks have off-the-shelf sparse embedding modules, most of them lack efficient support of distributed sparse update. DistDGL's KVStore shards the vertex embeddings in the same way as vertex features. Upon receiving the embedding gradients (via the PUSH interface), KVStore updates the embedding based on the optimizer the user registered.

\section{Distributed Sampler}

DGL has provided a set of flexible Python APIs to support a variety of sampling algorithms proposed in the literature. DistDGL keeps this API design but with a different internal implementation. At the beginning of each iteration, the trainer issues sampling requests using the target vertices in the current mini-batch. The requests are dispatched to the machines according to the core vertex assignment produced by the graph partitioning algorithm. Upon receiving the request, sampler servers call DGL's sampling operators on the local partition and transmit the result back to the trainer process. Finally, the trainer collects the results and stitches them together to generate a mini-batch.

DistDGL deploys multiple optimizations to effectively accelerate mini-batch generation. DistDGL can create multiple sampling worker processes for each trainer to sample minibatches in parallel. By issuing sampling requests to the sam- pling workers, trainers overlap the sampling cost with minibatch training. When a sampling request goes to the local sampler server, the sampling workers to access the graph structure stored on the local sampler server directly via shared memory to avoid the cost of the RPC stack. The sampling workers also overlaps the remote RPCs with local sampling computation by first issuing remote requests asynchronously. This effectively hides the network latency because the local sampling usually accounts for most of the sampling time. When a sampler server receives sampling requests, it only needs to sample vertices and edges from the local partition because our graph partitioning strategy (Section III-B) guarantees that the core vertices in a partition have the access to the entire neighborhood.

\section{E. Mini-batch Trainer}

Mini-batch trainers run on each machine to jointly estimate gradients and update parameters of users' models. DistDGL provides utility functions to split the training set distributedly and generate balanced workloads between trainers.

Each trainer samples data points uniformly at random to generate mini-batches independently. Because DistDGL generates balanced partitions (each partition has roughly the same number of nodes and edges) and uses synchronous SGD to train the model, the data points sampled collectively by all trainers in each iteration are still sampled uniformly at random across the entire dataset. As such, distributed training in DistDGL in theory does not affect the convergence rate or the model accuracy.

To balance the computation in each trainer, DistDGL uses a two-level strategy to split the training set evenly across all trainers at the beginning of distributed training. We first ensure that each trainer has the same number of training samples. The multi-constraint algorithm in METIS (Section III-B can only assign roughly the same number of training samples (vertices or edges) to each partition (as shown by the rectangular boxes on the top in Figure 5). We thus evenly split the training samples based on their IDs and assign the ID range to a machine whose graph partition has the largest overlap with the ID range. This is possible because we relabel vertex and edge IDs during graph partitioning and the vertices and edges in a partition have a contiguous ID range. There is a small misalignment between the training samples assigned to a trainer and the ones that reside in a partition. Essentially, we make a tradeoff between load balancing and data locality. In practice, as long as the graph partition algorithm balances the number of training samples between partitions, the tradeoff is negligible. If there are multiple trainers on one partition, we further split the local training vertices evenly and assign them to the trainers in the local machine. We find that random split in practice gives a fairly balanced workload assignment.

In terms of parameter synchronization, we use synchronous SGD to update dense model parameters. Synchronous SGD is commonly used to train deep neural network models and usually leads to better model accuracy. We use asynchronous SGD to update the sparse vertex embeddings in the Hogwild 


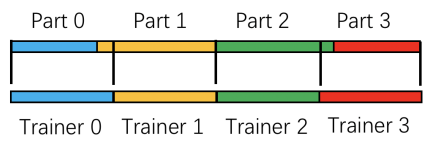

Fig. 5: Split the workloads evenly to balance the computation among trainer processes.

TABLE I: Dataset statistics from the Open Graph Benchmark [33].

\begin{tabular}{lrrr}
\hline Dataset & \# Nodes & \# Edges & Node Features \\
\hline OGBN-PRODUCT & $2,449,029$ & $61,859,140$ & 100 \\
OGBN-PAPERS 100M & $111,059,956$ & $3,231,371,744$ & 128 \\
\hline
\end{tabular}

fashion [32] to overlap communication and computation. In a large graph, there are many vertex embeddings. Asynchronous SGD updates some of the embeddings in a minibatch. Concurrent updates from multiple trainers rarely result in conflicts because mini-batches from different trainers run on different embeddings. Previous study [31] has verified that asynchronous update of sparse embeddings can significantly speed up the training with nearly no accuracy loss.

For distributed CPU training, DistDGL parallelizes the computation with both multiprocessing and multithreading. Inside a trainer process, we use OpenMP to parallelize the framework operator computation (e.g., sparse matrix multiplication and dense matrix multiplication). We run multiple trainer processes on each machine to parallelize the computation for nonuniform memory architecture (NUMA), which is a typical architecture for large CPU machines. This hybrid approach is potentially more advantageous than the multiprocessing approach for synchronous SGD because we need to aggregate gradients of model parameters from all trainer processes and broadcast new model parameters to all trainers. More trainer processes result in more communication overhead for model parameter updates.

\section{Evaluation}

In this section, we evaluate DistDGL to answer the following questions:

- Can DistDGL train GNNs on large-scale graphs and accelerate the training with more machines?

- Can DistDGL's techniques effectively increase the data locality for GNN training?

- Can our load balancing strategies effectively balance the workloads in the cluster of machines?

We focused on the node classification task using GNNs throughout the evaluation. The GNNs for other tasks such as link prediction mostly differ in the objective function while sharing most of the GNN architectures so we omit them in the experiments.

We benchmark the state-of-the-art GraphSAGE [2] model on two Open Graph Benchmark (OGB) datasets [33] shown in TableI. The GraphSAGE model has three layers of hidden size 256; the sampling fan-outs of each layer are 15, 10 and 5. We

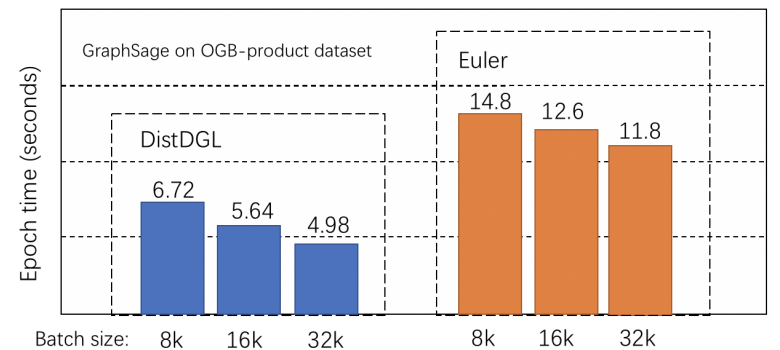

(a) The overall runtime per epoch with different global batch sizes.

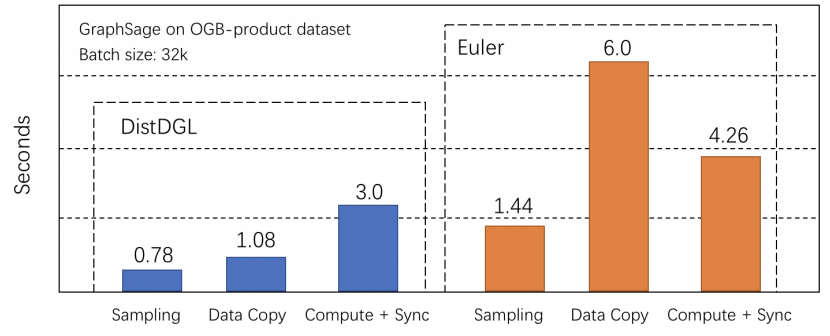

(b) The breakdown of epoch runtime for the batch size of $32 \mathrm{~K}$.

Fig. 6: DistDGL vs Euler on OGBN-PRODUCT graph on four m5n.24xlarge instances.

use a cluster of eight AWS EC2 m5n.24xlarge instances (96 VCPU, 384GB RAM each) connected by a 100Gbps network.

In all experiments, we use DGL v0.5 and Pytorch 1.5. For Euler experiments, we use Euler v2.0 and TensorFlow 1.12.

\section{A. DistDGL vs. other distributed GNN frameworks}

We compare the training speed of DistDGL with Euler [17], one of the state-of-the-art distributed GNN training frameworks, on four m5n.24xlarge instances. Euler is designed for distributed mini-batch training, but it adopts different parallelization strategy from DistDGL. It parallelizes computation completely with multiprocessing and uses one thread for both forward and backward computation as well as sampling inside a trainer. To have a fair comparison between the two frameworks, we run mini-batch training with the same global batch size (the total size of the batches of all trainers in an iteration) on both frameworks because we use synchronized SGD to train models.

DistDGL gets $2.2 \times$ speedup over Euler in all different batch sizes (Figure 6a). To have a better understanding of DistDGL's performance advantage, we break down the runtime of each component within an iteration shown in Figure 6b. The main advantage of DistDGL is data copy, in which DistDGL has more than $5 \times$ speedup. This is expected because DistDGL uses METIS to generate partitions with minimal edge cuts and trainers are co-located with the partition data to reduce network communication. The speed of data copy in DistDGL gets close to local memory copy while Euler has to copy data through TCP/IP from the network. DistDGL also has $2 \times$ speedup in sampling over Euler for the same reason: DistDGL samples majority of vertices and edges from the local 


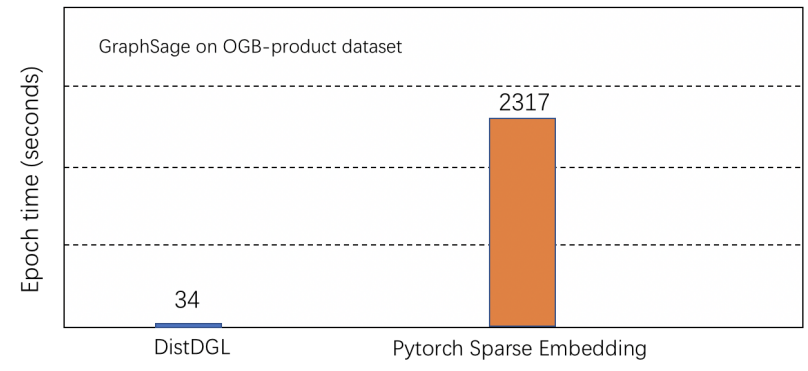

Fig. 7: The GraphSage model with DistDGL's and Pyotch's sparse Embedding on the OGBN-PRODUCT graph.

partition to generate mini-batches. DistDGL relies on DGL and Pytorch to perform sparse and dense tensor computation in a mini-batch and uses Pytorch to synchronize gradients among trainers while Euler relies on TensorFlow for both mini-batch computation and gradient synchronization. DistDGL is slightly faster in mini-batch computation and gradient synchronization. Unfortunately, we cannot separate the batch computation and gradient synchronization in Pytorch.

\section{B. DistDGL's sparse embedding vs. Pytorch's sparse embed- ding}

Many graph datasets do not have vertex features. We typically use transductive GNN models with learnable vertex embeddings for these graphs. DistDGL provides distributed embeddings for such use case, with optimizations for sparse updates. Deep learning frameworks, such as Pytorch, also provide the sparse embedding layer for similar use cases and the embedding layer can be trained in a distributed fashion. To evaluate the efficiency of DistDGL's distributed embeddings, we adapt the GraphSage model by replacing vertex data of the input graph with DistDGL's or Pytorch's sparse embeddings.

The GraphSage model with DistDGL's sparse embeddings on OGBN-PRODUCT graph gets almsot $70 \times$ speedup over the version with Pytorch sparse embeddings (Figure 7). The main difference is that DistDGL's sparse embeddings are updated with DistDGL's efficient KVStore, which is natural for implementing sparse embedding updates. As such, it gets all benefits of DistDGL's optimizations, such as co-location of data and computation. In contrast, Pytorch's sparse embeddings are updated with its DistributedDataParallel module. Essentially, it is implemented with the AllReduce primitive, which requires the gradient tensor exchanged between trainers to have exactly the same shape. As such, Pytorch has to pad the gradient tensor of sparse embeddings to the same size.

\section{Scalability}

We further evaluate the scalability of DistDGL in the EC2 cluster. In this experiment, we fix the mini-batch size in each trainer and increase the number of trainers when the number of machines increases. We use the batch size of 2000 per trainer.

DistDGL achieves a linear speedup as the number of machines increases in the cluster (Figure 8) for both OGB
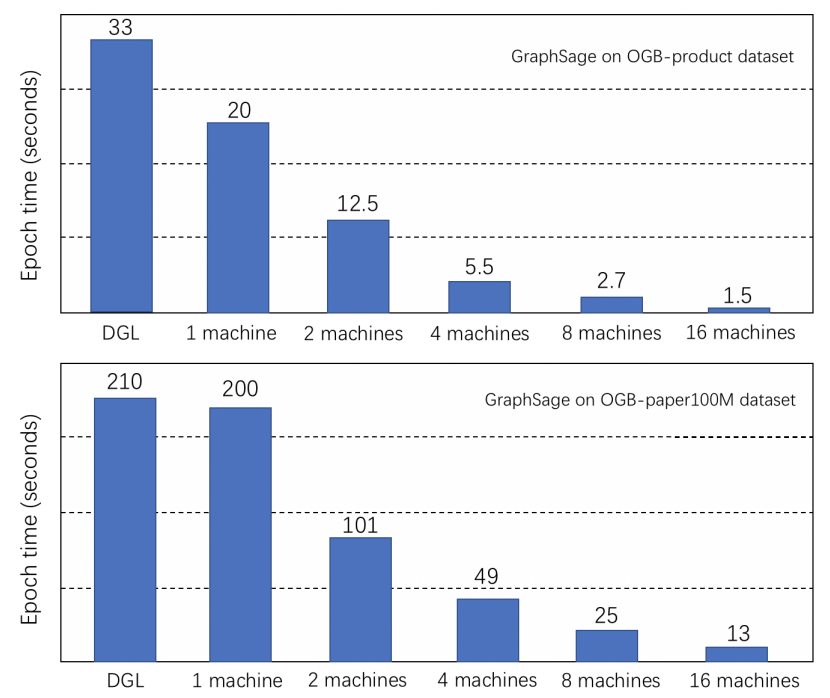

Fig. 8: DistDGL achieves linear speedup w.r.t. the number of machines.

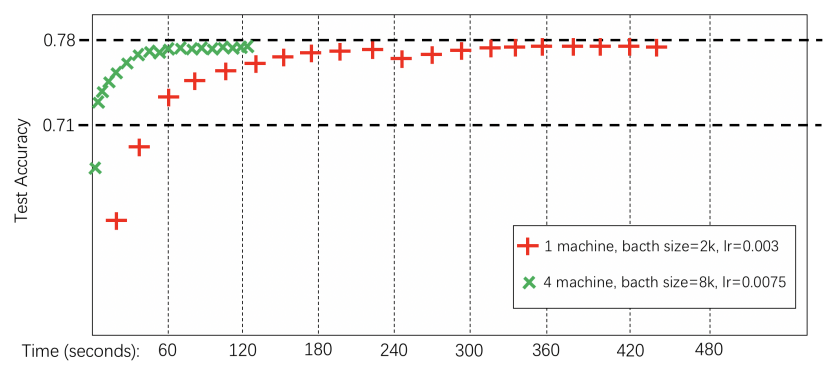

Fig. 9: DistDGL convergence of distributed training.

datasets. When running on a larger cluster, DistDGL needs to perform more sampling on remote machines and fetch more data from remote machines. This linear speedup indicates that our optimizations prevent network communication from being the bottleneck. It also suggests that the system is well balanced when the number of machines increases. With all of our optimizations, DistDGL can easily scale to large graphs with hundreds of millions of nodes. It takes only 13 seconds to train the GraphSage model on the OGBN-PAPERS 100M graph in a cluster of $16 \mathrm{~m} 5.24 x$ large machines.

We also compare DistDGL with DGL's multiprocessing training (two trainer processes). DistDGL running on a single machine with two trainers outperforms DGL. This may attribute to the different multiprocessing sampling used by the two frameworks. DGL relies on Pytroch dataloader's multiprocessing to sample mini-batches while DistDGL uses dedicated sampler processes to generate mini-batches.

In addition to the training speed, we also verify the training accuracy of DistDGL on different numbers of machines (Figure 9). We can see that DistDGL quickly converges to almost the same peak accuracy achieved by the single-machine training, which takes a much longer time to converge. 

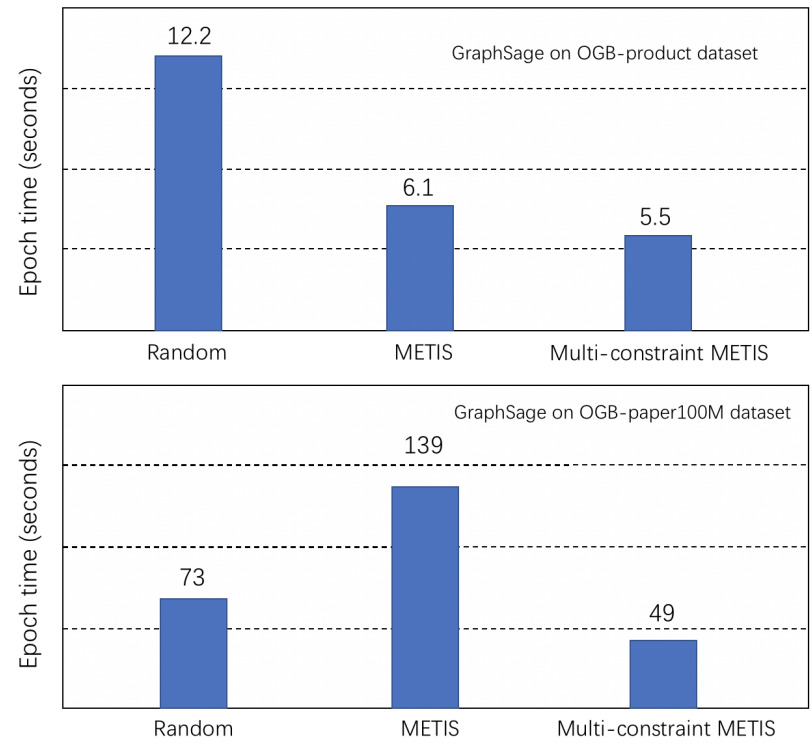

Fig. 10: METIS vs Random Partition on four machines

\section{Ablation Study}

We further study the effectiveness of the main optimizations in DistDGL: 1) reducing network traffic by METIS graph partitioning and co-locating data and computation, 2) balance the graph partitions with multi-constraint partitioning. To evaluate their effectiveness, we compare DistDGL's graph partitioning algorithm with two alternatives: random graph partitioning and default METIS partitioning without multi-constraints. We use a cluster of four machines to run the experiments.

METIS partitioning with multi-constraints to balance the partitions achieves good performance on both datasets (Figure 10. Default METIS partitioning performs well compared with random partitioning $(2.14 \times$ speedup) on the OGBNPRODUCT graph due to its superior reduction of network communication; adding multiple constraints to balance partitions gives additional $4 \%$ improvement over default METIS partitioning. However, default METIS partitioning achieves much worse performance than random partitioning on the OGBNPAPERS 100M graph due to high imbalance between partitions created by METIS, even though METIS can effectively reduce the number of edge cuts between partitions. Adding multiconstraint optimizations to balance the partitions, we see the benefit of reducing network communication. This suggests that achieving load balancing is as important as reducing network communication for improving performance.

\section{RELATED WORK}

\section{A. Distributed DNN Training}

There are many system-related works to optimize distributed deep neural network (DNN) training. The parameter server [34] is designed to maintain and update the sparse model parameters. Horovod [35] and Pytorch distributed [27] uses allreduce to aggregate dense model parameters but does not work for sparse model parameters. BytePs [20] adopts more sophisticated techniques of overlapping model computation and gradient communication to accelerate dense model parameter updates. Many works reduces the amount of communication by using quantization [36] or sketching [37]. Several recent work focuses on relaxing the synchronization of weights [38], [39] in case some workers run slower than others temporally due to some hardware issues. GNN models are composed of multiple operators organized into multiple graph convolution network layers shared among all nodes and edges. Thus, GNN training also has dense parameter updates. However, the network traffic generated by dense parameter updates is relatively small compared with node/edge features. Thus, reducing the network traffic of dense parameter updates is not our main focus for distributed GNN training.

\section{B. Distributed GNN Training}

A few works have been developed to scale GNN training on large graph data in the multi-GPU setting or distributed setting. Some of them [12]-[14] perform full graph training on multiple GPUs or distributed memory whose aggregated memory fit the graph data. However, we believe full graph training is an inefficient way to train a GNN model in a large graph data because one model update requires significant amount of computation. The mini-batch training has been widely adopted in training a neural network.

Multiple GNN frameworks [15]-[17] built by industry adopt distributed mini-batch training. However, none of these frameworks adopt locality-aware graph partitioning and colocate data and communication. As shown in our experiment, reducing communication is a key to achieve good performance.

\section{Distributed graph processing}

There are many works on distributed graph processing frameworks. Pregel [7] is one of the first frameworks that adopt message passing and vertex-centric interface to perform basic graph analytics algorithms such as breadth-first search and triangle counting. PowerGraph [8] adopts vertex cut for graph partitioning and gather-and-scatter interface for computation. PowerGraph had significant performance improvement overhead Pregel. Gemini [30] shows that previous distributed graph processing framework has significant overhead in a single machine. It adopts the approach to improve graph computation in a single machine first before optimizing for distributed computation. Even though the computation pattern of distributed mini-batch training of GNN is very different from traditional graph analytics algorithms, the evolution of graph processing frameworks provide valuable lessons for us and many of the general ideas, such as locality-aware graph partitioning and co-locating data and computation, are borrowed to optimize distributed GNN training.

\section{CONCLUSION}

We develop DistDGL for distributed GNN training. We adopt Metis partitioning to generate graph partitions with minimum edge cuts and co-locate data and computation to reduce the network communication. We deploy multiple 
strategies to balance the graph partitions and mini-batches generated from each partition. We demonstrate that achieving high training speed requires both network communication reduction and load balancing. Our experiments show DistDGL has linear speedup of training GNN models on a cluster of CPU machines without compromising model accuracy.

\section{REFERENCES}

[1] Google, "Freebase data dumps," https://developers.google.com/freebase/ data

[2] W. L. Hamilton, R. Ying, and J. Leskovec, "Inductive representation learning on large graphs," in Proceedings of the 31st International Conference on Neural Information Processing Systems, ser. NIPS'17, 2017, p. 1025-1035.

[3] J. Chen, J. Zhu, and L. Song, "Stochastic training of graph convolutional networks with variance reduction," ser. Proceedings of Machine Learning Research, J. Dy and A. Krause, Eds., vol. 80. Stockholmsmässan, Stockholm Sweden: PMLR, 10-15 Jul 2018, pp. 942-950.

[4] J. Chen, T. Ma, and C. Xiao, "FastGCN: Fast learning with graph convolutional networks via importance sampling," in International Conference on Learning Representations, 2018.

[5] W. Huang, T. Zhang, Y. Rong, and J. Huang, "Adaptive sampling towards fast graph representation learning," CoRR, vol. abs/1809.05343, 2018.

[6] R. Ying, R. He, K. Chen, P. Eksombatchai, W. L. Hamilton, and J. Leskovec, "Graph convolutional neural networks for web-scale recommender systems," CoRR, vol. abs/1806.01973, 2018.

[7] G. Malewicz, M. H. Austern, A. J. Bik, J. C. Dehnert, I. Horn, N. Leiser, and G. Czajkowski, "Pregel: A system for large-scale graph processing," in Proceedings of the 2010 ACM SIGMOD International Conference on Management of Data, ser. SIGMOD '10, New York, NY, USA, 2010, p. $135-146$.

[8] J. E. Gonzalez, Y. Low, H. Gu, D. Bickson, and C. Guestrin, "Powergraph: Distributed graph-parallel computation on natural graphs," in 10th USENIX Symposium on Operating Systems Design and Implementation (OSDI 12), Nov. 2012.

[9] J. Shun and G. E. Blelloch, "Ligra: A lightweight graph processing framework for shared memory," SIGPLAN Not., vol. 48, no. 8, p. 135-146, Feb. 2013.

[10] M. Wang, D. Zheng, Z. Ye, Q. Gan, M. Li, X. Song, J. Zhou, C. Ma, L. Yu, Y. Gai, T. Xiao, T. He, G. Karypis, J. Li, and Z. Zhang, "Deep graph library: A graph-centric, highly-performant package for graph neural networks," arXiv preprint arXiv:1909.01315, 2019.

[11] M. Fey and J. E. Lenssen, "Fast graph representation learning with pytorch geometric," arXiv preprint arXiv:1903.02428, 2019.

[12] Z. Jia, S. Lin, M. Gao, M. Zaharia, and A. Aiken, "Improving the accuracy, scalability, and performance of graph neural networks with roc," in Proceedings of Machine Learning and Systems, I. Dhillon, D. Papailiopoulos, and V. Sze, Eds., 2020, vol. 2, pp. 187-198.

[13] L. Ma, Z. Yang, Y. Miao, J. Xue, M. Wu, L. Zhou, and Y. Dai, "Neugraph: Parallel deep neural network computation on large graphs," in 2019 USENIX Annual Technical Conference (USENIX ATC 19), Renton, WA, Jul. 2019, pp. 443-458.

[14] A. Tripathy, K. Yelick, and A. Buluc, "Reducing communication in graph neural network training," arXiv preprint arXiv:2005.03300, 2020.

[15] R. Zhu, K. Zhao, H. Yang, W. Lin, C. Zhou, B. Ai, Y. Li, and J. Zhou, "AliGraph: A comprehensive graph neural network platform," arXiv preprint arXiv:1902.08730, 2019.

[16] D. Zhang, X. Huang, Z. Liu, Z. Hu, X. Song, Z. Ge, Z. Zhang, L. Wang, J. Zhou, Y. Shuang, and Y. Qi, "AGL: a scalable system for industrialpurpose graph machine learning," arXiv preprint arXiv:2003.02454, 2020.

[17] "Euler github," https://github.com/alibaba/euler 2020

[18] M. Li, D. G. Andersen, J. W. Park, A. J. Smola, A. Ahmed, V. Josifovski, J. Long, E. J. Shekita, and B.-Y. Su, "Scaling distributed machine learning with the parameter server," in Proceedings of the 11th USENIX Conference on Operating Systems Design and Implementation, ser. OSDI'14. USA: USENIX Association, 2014, p. 583-598.

[19] T. Chilimbi, Y. Suzue, J. Apacible, and K. Kalyanaraman, "Project adam: Building an efficient and scalable deep learning training system," in 11th USENIX Symposium on Operating Systems Design and Implementation (OSDI 14), Broomfield, CO, Oct. 2014, pp. 571-582.
[20] Y. Peng, Y. Zhu, Y. Chen, Y. Bao, B. Yi, C. Lan, C. Wu, and C. Guo, "A generic communication scheduler for distributed dnn training acceleration," in Proceedings of the 27th ACM Symposium on Operating Systems Principles, ser. SOSP '19, New York, NY, USA, 2019, p. 16-29.

[21] G. Karypis and V. Kumar, "A fast and high quality multilevel scheme for partitioning irregular graphs," SIAM Journal on Scientific Computing, vol. 20, no. 1, pp. 359-392, 1998.

[22] J. Gilmer, S. S. Schoenholz, P. F. Riley, O. Vinyals, and G. E. Dahl, "Neural message passing for quantum chemistry," in Proceedings of the 34th International Conference on Machine Learning - Volume 70, 2017.

[23] P. W. Battaglia, J. B. Hamrick, V. Bapst, A. Sanchez-Gonzalez, V. Zambaldi, M. Malinowski, A. Tacchetti, D. Raposo, A. Santoro, R. Faulkner et al., "Relational inductive biases, deep learning, and graph networks," arXiv preprint arXiv:1806.01261, 2018.

[24] D. Zou, Z. Hu, Y. Wang, S. Jiang, Y. Sun, and Q. Gu, "Layer-dependent importance sampling for training deep and large graph convolutional networks," arXiv preprint arXiv:1911.07323, 2019.

[25] W. Huang, T. Zhang, Y. Rong, and J. Huang, "Adaptive sampling towards fast graph representation learning," arXiv preprint arXiv:1809.05343, 2018.

[26] W.-L. Chiang, X. Liu, S. Si, Y. Li, S. Bengio, and C.-J. Hsieh, "Clustergcn," Proceedings of the 25th ACM SIGKDD International Conference on Knowledge Discovery \& Data Mining, Jul 2019.

[27] S. Li, Y. Zhao, R. Varma, O. Salpekar, P. Noordhuis, T. Li, A. Paszke, J. Smith, B. Vaughan, P. Damania et al., "Pytorch distributed: Experiences on accelerating data parallel training," arXiv preprint arXiv:2006.15704, 2020.

[28] S. Eyerman, W. Heirman, K. D. Bois, J. B. Fryman, and I. Hur, "Manycore graph workload analysis," in Proceedings of the International Conference for High Performance Computing, Networking, Storage, and Analysis, ser. SC '18. IEEE Press, 2018.

[29] G. Karypis and V. Kumar, "Multilevel algorithms for multi-constraint graph partitioning," in Proceedings of the 1998 ACM/IEEE Conference on Supercomputing, USA, 1998, p. 1-13.

[30] X. Zhu, W. Chen, W. Zheng, and X. Ma, "Gemini: A computationcentric distributed graph processing system," in 12th USENIX Symposium on Operating Systems Design and Implementation (OSDI 16), Nov. 2016.

[31] D. Zheng, X. Song, C. Ma, Z. Tan, Z. Ye, J. Dong, H. Xiong, Z. Zhang, and G. Karypis, "DGL-KE: Training knowledge graph embeddings at scale," arXiv preprint arXiv:2004.08532, 2020.

[32] F. Niu, B. Recht, C. Re, and S. J. Wright, "Hogwild!: A lock-free approach to parallelizing stochastic gradient descent," arXiv preprint arXiv:1106.5730, 2011.

[33] W. Hu, M. Fey, M. Zitnik, Y. Dong, H. Ren, B. Liu, M. Catasta, and J. Leskovec, "Open graph benchmark: Datasets for machine learning on graphs," arXiv preprint arXiv:2005.00687, 2020.

[34] M. Li, D. G. Andersen, J. W. Park, A. J. Smola, A. Ahmed, V. Josifovski, J. Long, E. J. Shekita, and B.-Y. Su, "Scaling distributed machine learning with the parameter server," in 11th $\{$ USENIX $\}$ Symposium on Operating Systems Design and Implementation ( $\{$ OSDI\} 14), 2014, pp. $583-598$.

[35] A. Sergeev and M. D. Balso, "Horovod: fast and easy distributed deep learning in tensorflow," arXiv preprint arXiv:1802.05799, 2018.

[36] F. Seide, H. Fu, J. Droppo, G. Li, and D. Yu, "1-bit stochastic gradient descent and its application to data-parallel distributed training of speech dnns," in Fifteenth Annual Conference of the International Speech Communication Association, 2014.

[37] N. Ivkin, D. Rothchild, E. Ullah, V. Braverman, I. Stoica, and R. Arora, "Communication-efficient distributed SGD with sketching," arXiv preprint arXiv:1903.04488, 2019.

[38] Q. Ho, J. Cipar, H. Cui, S. Lee, J. K. Kim, P. B. Gibbons, G. A. Gibson, G. Ganger, and E. P. Xing, "More effective distributed $\mathrm{ml}$ via a stale synchronous parallel parameter server," in Advances in neural information processing systems, 2013, pp. 1223-1231.

[39] Q. Luo, J. Lin, Y. Zhuo, and X. Qian, "Hop: Heterogeneity-aware decentralized training," in Proceedings of the Twenty-Fourth International Conference on Architectural Support for Programming Languages and Operating Systems, 2019, pp. 893-907. 